\title{
Modelo de elementos finitos de la columna lumbar
}

\author{
F. EZQUERRO JUANCO, A. SIMÓN MATA, \\ E. MELLADO ARJONA, F. VILLANUEVA PAREJA* \\ Departamento de Ingeniería Mecánica y Energética. \\ E.T.S.I.I. Universidad de Málaga. \\ *Unidad de Columna del Servicio de Cirugía Ortopédica y Traumatología \\ Hospital Regional de Málaga
}

\begin{abstract}
Resumen
En este trabajo se describe un modelo de Elementos Finitos de la columna lumbar humana. El objetivo buscado es la utilización del mismo como herramienta de investigación aplicada a la cirugía ortopédica de columna lumbar. Para conseguir este objetivo se ha elaborado un modelo no lineal y paramétrico de la columna lumbar completa, el cual puede modificarse con facilidad tanto en su geometría como en sus características mecánicas de modo que puedan reflejarse tanto distintas alteraciones segmentarias como diversas técnicas de fijación. También se expone la contrastación del modelo, realizada a partir de resultados experimentales recogidos de la literatura sobre el tema.
\end{abstract}

Palabras clave: Análisis biomecánico, Modelado por elementos finitos, Columna lumbar.

\section{Introducción}

Las técnicas de tratamiento quirúrgico encaminadas a la corrección y estabilización de los segmentos raquídeos alterados mediante fijación transpedicular han mejorado considerablemente el pronóstico de muchos casos que con anterioridad no habrían tenido una solución quirúrgica aceptable. Cada nuevo avance en estas técnicas ha debido basarse en la experiencia acumulada con técnicas anteriores y en el diseño por tanteo y apreciaciones personales de su autor, que generalmente empieza por introducir con cautela modificaciones a procedimientos ya consagrados, aprovechando casos peculiares no bien resueltos por la cirugía convencional y en los que es por tanto éticamente aceptable ensayar una nueva idea.

La existencia de un modelo biomecánico que permita simular el comportamiento de la columna vertebral humana sería de gran utilidad, ya que podría ser utilizado como una herramienta para el estudio de las técnicas quirúrgicas empleadas, el diseño de implantes y la ejecución de pruebas biomecánicas previas a su experimentación clínica.

Con el fin de conseguir estos objetivos se ha desarrollado un modelo no lineal de elementos finitos de la columna lumbar humana. Los requisitos exigidos a este modelo son: a) representar fielmente el comportamiento mecánico de los distintos componentes de la columna lumbar humana, b) Capacidad para personalizar su geometría en función de una serie de parámetros que puedan ser medidos en radiografías $\mathrm{y}$ tomografías (diámetros vertebrales, altura de discos, etc.) y c) capacidad de ser modificado de modo que puedan reflejarse tanto distintas alteraciones segmentarias como diversas técnicas y aparatos de fijación.

El método de los elementos finitos es, probablemente, la técnica mas utilizada para modelar el comportamiento mecánico de los segmentos vertebrales. Los primeros modelos fueron bidimensionales ${ }^{1}$, existiendo en la actualidad varios modelos tridimensionales no lineales de 
segmentos aislados de la columna lumbar ${ }^{2,3}$, así como de la columna lumbar completa ${ }^{4,5}$ El modelo de Lavaste es paramétrico, construyéndose toda la geometría a partir de una serie de parámetros medibles con radiografía biplanar. Este mismo principio se utiliza en la construcción del modelo presentado, de forma que pueda reproducir cualquier particularidad geométrica e incluso permita la planificación individualizada de las técnicas quirúrgicas estudiadas.

\section{Materiales y métodos}

\section{Modelo de elementos finitos}

Para la construcción del modelo se ha utilizado el módulo no lineal del paquete comercial de elementos finitos COSMOS/M.

La geometría del modelo, que reproduce la columna lumbar completa, se construye a partir de una serie de parámetros medibles sobre radiografías y/o tomografías axiales de la columna lumbar, los parámetros correspondientes al cuerpo vertebral y al disco aparecen esquematizados en la Figura 1.

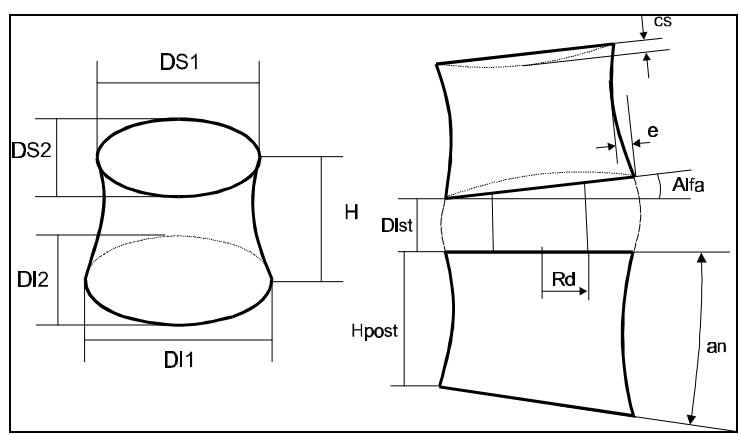

Figura 1. Parámetros geométricos del cuerpo vertebral y el disco.

Los tipos de elementos utilizados así como las propiedades de material asignadas a los mismos aparecen en la Tabla 1, habiéndose obtenido de la literatura $^{6,7,8}$.En la Figura 2 puede apreciarse una perspectiva del modelo.

Para modelar el hueso se han utilizado elementos sólidos homogéneos de 8 nudos, con una rigidez intermedia entre las correspondientes al hueso trabecular y al cortical. Al no diferenciar entre estos dos tipos de hueso la distribución de tensiones en las vértebras, obtenida por el modelo, no será exacta, sin embargo no cabe esperar grandes diferencias en el comportamiento global de los segmentos, debido a la alta rigidez del hueso respecto al resto de componentes.

Tabla 1. Propiedades de los materiales utilizados.

\begin{tabular}{l|ccc} 
& $\begin{array}{c}\text { Tipo de } \\
\text { elemento }\end{array}$ & $\begin{array}{c}\text { Mód. de } \\
\text { Young } \\
\text { (MPa) }\end{array}$ & $\begin{array}{c}\text { Módulo de } \\
\text { Poisson }\end{array}$ \\
\hline Hueso & SOLID & 3500 & 0.25 \\
$\begin{array}{l}\text { Núcleo } \\
\text { pulposo }\end{array}$ & SOLID & 0.13 & 0.49998 \\
$\begin{array}{l}\text { Materia base } \\
\text { del anillo } \\
\text { fibroso }\end{array}$ & SOLID & 4.2 & 0.45 \\
$\begin{array}{l}\text { Fibras } \\
\text { colágenas }\end{array}$ & TRUSS3D & & \\
$\begin{array}{l}\text { Ligamentos } \\
\text { Contacto en } \\
\text { carillas }\end{array}$ & TRUSS3D & & \\
& GAP & \\
\end{tabular}

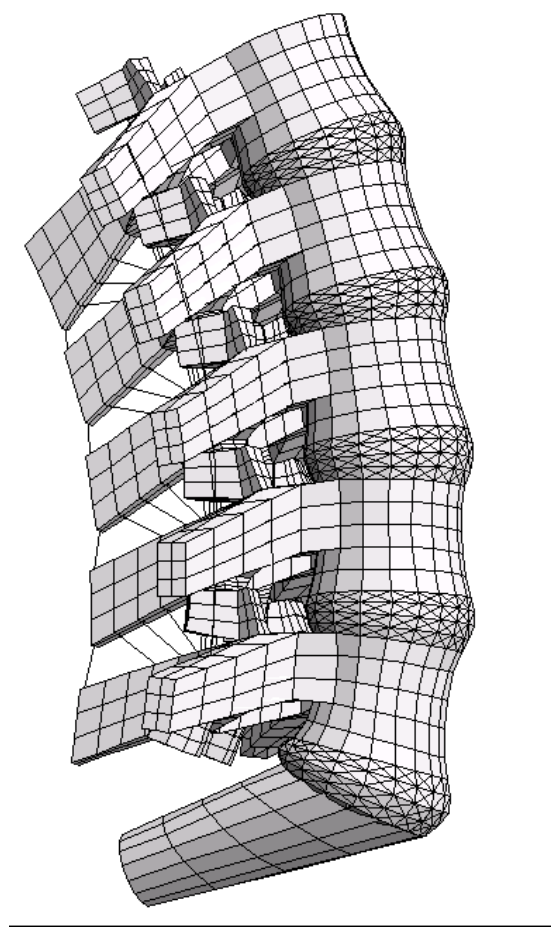

Figura 2. Modelo de elementos finitos

En el modelo de los discos intervertebrales se ha diferenciado entre núcleo y anillo fibroso. El primero ha sido modelado con elementos sólidos de 8 nudos con bajo módulo de elasticidad y coeficiente de Poisson cercano a 0.5 (Tabla 1) de modo que su comportamiento sea similar a un 
fluido incompresible de módulo de elasticidad volumétrico igual al encontrado en la literatura 1666.7 MPa. El anillo fibroso se ha simulado como un material compuesto constituido por fibras embebidas en una matriz elástica de módulo de Poisson igual a $0.45^{2,3}$. Para modelar la matriz se han utilizado elementos sólidos y para las fibras colágenas elementos cable (resistentes solo a tracción) con propiedades de material elástico nolineal obtenidas de la literatura.

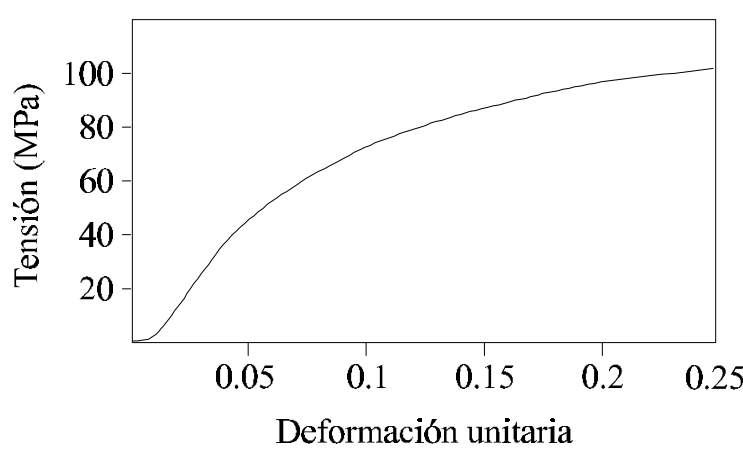

Figura 3. Curva tensión-deformación utilizada para las fibras colágenas

La orientación de estos elementos cable (fibras) en el modelo depende del número de elementos utilizados para modelar la matriz del anillo así como del tamaño del disco, por tanto se ha elegido el número de elementos de la matriz, en dirección axial, de forma que la inclinación media de las fibras respecto al plano medio del disco intervertebral, para un disco lumbar de dimensiones comunes, sea de $30^{\circ}{ }^{7}$. Las fibras se han distribuido en dos capas concéntricas incluyendo en cada capa dos haces de fibras cruzadas, formando entre ellas un ángulo medio de $120^{\circ}$. La sección transversal dada a estos elementos se ha elegido de modo que el contenido total, en volumen, de fibra sea el $16 \%$ del correspondiente al anillo fibroso ${ }^{2}$.

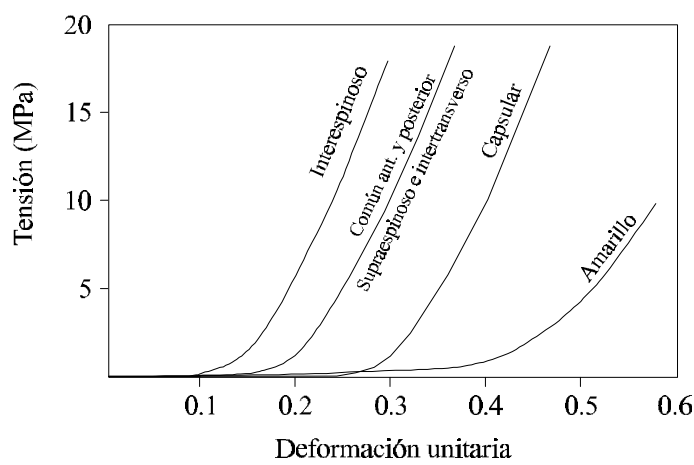

Figura 4. Curva tensión-deformación utilizada para los ligamentos
Para los ligamentos se han utilizado, al igual que en el caso de las fibras, elementos cable con propiedades de material elástico no lineal ${ }^{2}$. Mientras que el contacto entre las carillas articulares se ha modelado por medio de elementos GAP, colocados entre las superficies de cada par de carillas, los cuales permiten transmitir compresión en dirección perpendicular al contacto entre ambas. La separación inicial entre carillas es un parámetro mas para la construcción del modelo, en las comprobaciones expuestas a continuación, se ha tomado, basándose en las medidas realizadas sobre tomografías, un valor de $0.5 \mathrm{~mm}$. igual para todos los niveles vertebrales.

\section{Análisis del modelo}

Para comprobar la bondad del modelo de elementos finitos se ha analizado la respuesta del mismo ante una serie de solicitaciones comunes en la columna lumbar. Los resultados obtenidos se han contrastado con valores publicados en la literatura por otros autores ${ }^{9,5,10,11,12,4}$. Las comprobaciones realizadas son las siguientes:

En primer lugar se ha comparado el comportamiento del modelo con resultados publicados de experimentos realizados sobre columnas cadavéricas, los datos utilizados se refieren al comportamiento ante distintas solicitaciones de distintos segmentos vertebrales lumbares aislados, por ello se ha aprovechado la propiedad de realizar alteraciones sobre el modelo original de la columna lumbar completa, para obtener un modelo de un segmento vertebral aislado, en concreto se ha utilizado el segmento L3L4. Las solicitaciones simuladas sobre este segmento son las siguientes: compresión, cortante, flexión, extensión, flexión lateral y torsión (Figura $5)$.

Las fuerzas (Compresión y Cortante) se han aplicado sobre el nudo central del cuerpo vertebral superior (L3), en la dirección correspondiente (axial, antero-posterior) de los ejes de referencia solidarios con dicho cuerpo vertebral. Los momentos respecto a estos mismos ejes se han introducido como pares de fuerzas aplicadas sobre nudos periféricos pertenecientes a la sección transversal media del cuerpo vertebral superior (L3). Las condiciones de contorno impuestas sobre el cuerpo vertebral inferior consisten en la fijación de los desplazamientos de los nudos pertenecientes a la placa limitante inferior y a las superficies de contacto de las carillas articulares superiores del siguiente cuerpo vertebral (L5). 


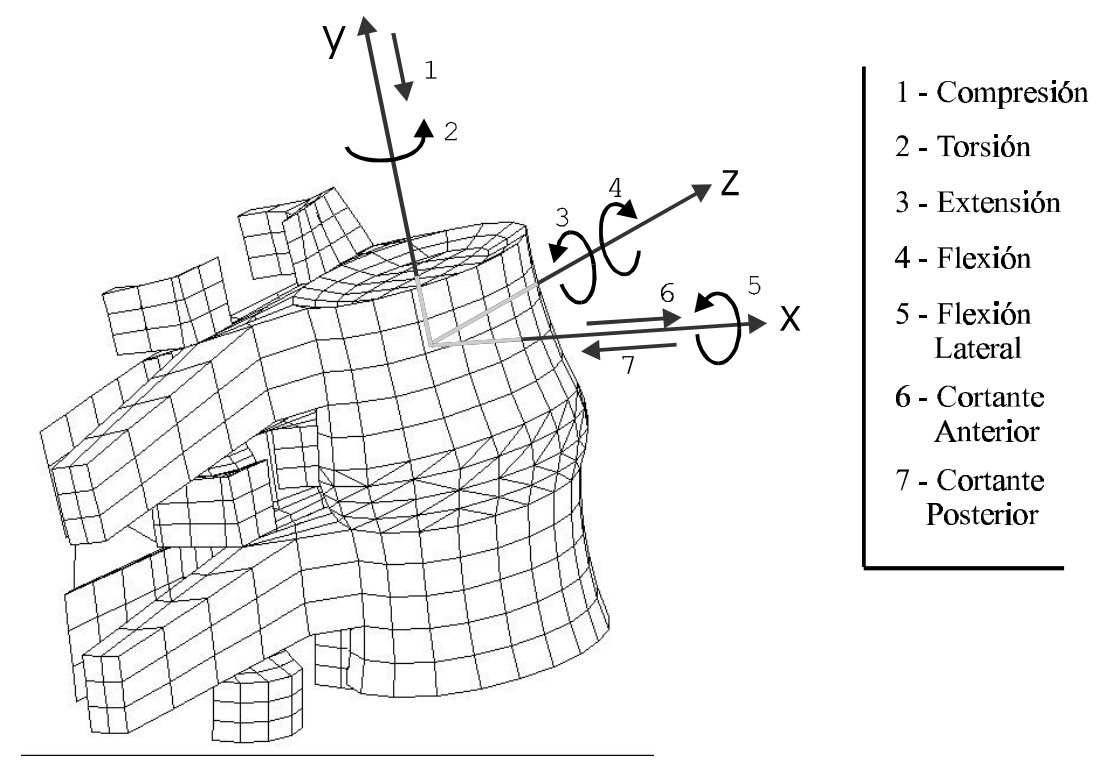

Figura 5. Solicitaciones consideradas sobre el segmento L3-L4

Las cargas han sido introducidas de modo incremental aumentando linealmente desde cero hasta su valor máximo en ocho etapas. Los valores máximos utilizados son los siguientes: Compresión: 2000 N; Cortante: 400 N; Torsión: 12 Nm; Flexión, extensión y flexión lateral $16 \mathrm{Nm}$.

En segundo lugar se ha establecido la comparación con los resultados obtenidos por otro modelo de elementos finitos suficientemente contrastado ${ }^{4}$. En concreto se han comparado los desplazamientos de los centros geométricos de los cinco cuerpos vertebrales cuando se somete a la columna lumbar completa a una compresión variable, en 28 etapas, entre 0 y $700 \mathrm{~N}$. Las cargas y condiciones de contorno corresponden con las utilizadas por Shirazi-Adl y cols ${ }^{4}$ :Carga aplicada sobre el centro del cuerpo vertebral L1 en la dirección de un eje ' $y$ ' perpendicular al plano medio del disco intervertebral L3-L4, sacro fijo en todas direcciones y centro del cuerpo vertebral L1 fijo en el plano perpendicular a dicho eje ' $y$ '.

Los parámetros geométricos utilizados en el modelo de partida para ambas comprobaciones son los valores medios para columnas lumbares encontrados por Panjabi y colaboradores ${ }^{13}$. Los valores de diámetro de cuerpos vertebrales, ángulo entre ellos y altura posterior del disco aparecen en la Tabla 2.
Tabla 2. Diámetro de los cuerpos vertebrales, ángulos y altrua posterior del disco.

\begin{tabular}{c|ccccccc} 
& DS1 & DS2 & DI1 & DI2 & Dist & Alfa & An \\
\hline L3 & 44.1 & 35.2 & 48 & 34.8 & & & 0 \\
L4 & 46.6 & 35.5 & 49.5 & 33.9 & & & 0
\end{tabular}

\section{Resultados y discusión}

\section{Segmento L3-L4}

En las gráficas de las Figuras 6 a 9 aparecen representados los desplazamientos del cuerpo vertebral superior (L3) frente a las fuerzas, o momentos, aplicadas. En los casos de compresión y cortante el valor representado corresponde al desplazamiento, axial y antero-posterior respectivamente, del nudo central del cuerpo vertebral superior. Para los casos de torsión, flexión, extensión y flexión lateral el movimiento reflejado en la gráfica corresponde al ángulo girado por el cuerpo vertebral superior en la misma dirección que la solicitación considerada en cada caso. Todos los desplazamientos y giros medidos están expresados en los ejes solidarios a la vértebra superior y son relativos al cuerpo vertebral inferior. 


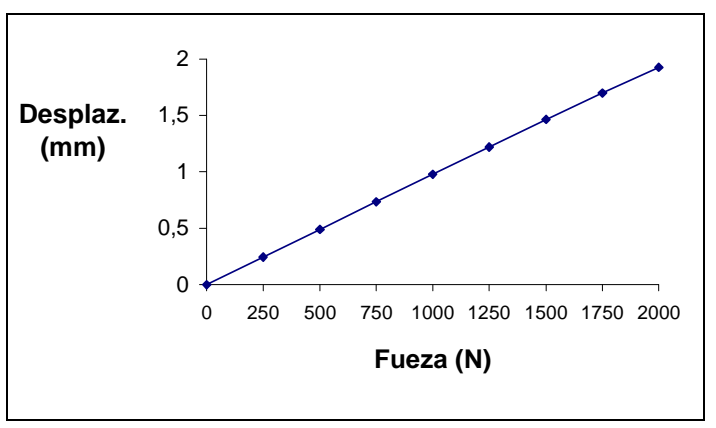

Figura. 6. Desplazamiento axial del centro de L3 vs Fuerza de compresión

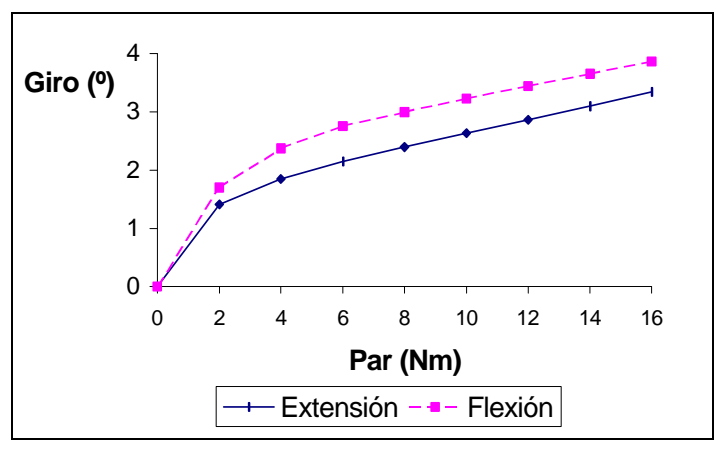

Figura. 8. Giro de L3 en el plano sagital vs Momento aplicado (Flexión/Extensión)

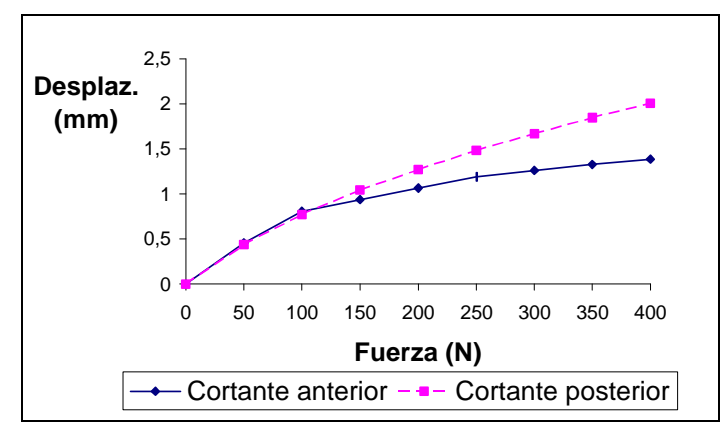

Figura. 7. Desplazamientos (valor absoluto) en dirección antero-posterior vs Fuerza cortante (anterior o posterior)

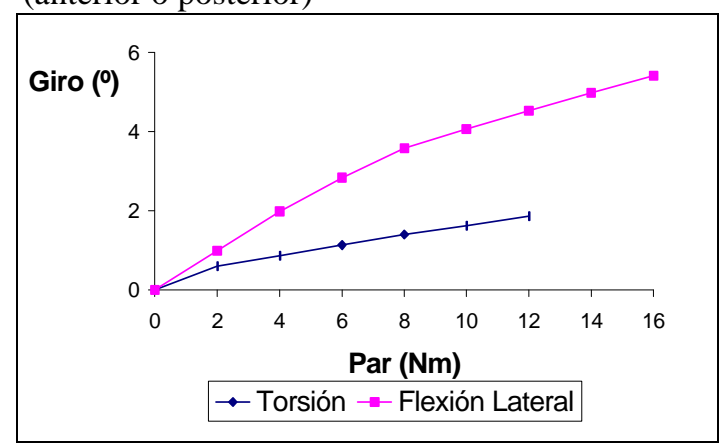

Figura. 9. Giro de L3 vs Momento aplicado (Torsión o Flexión lateral)

Tabla 3. Rigidez secante media de segmentos lumbares. Comparación de valores medios obtenidos experimentalmente por diversos autores y los obtenidos por el modelo de EE.FF. para el segmento L3L4.

\begin{tabular}{l|ccccc} 
& Schultz $^{9,1}$ & Markolf $^{1}$ & Panjabi $^{11^{1}}$ & Lavaste $^{5}$ & Modelo* $^{*}$ \\
\hline Compresión & 667 & 1000 & 1300 & 800 & 1100 \\
Cortante anterior & 145 & - & 125 & - & 123.4 \\
Cortante posterior & 143 & - & - & - & 129.1 \\
Torsión & 6.8 & 7.7 & 18.8 & 7 & 5.3 \\
Flexión & 0.9 & 2.7 & 1.7 & 3 & 2.2 \\
Extensión & 2.2 & 3.6 & 3 & - & 2.8 \\
Flexión lateral & 1.1 & 2 & 2.3 & 4 & 2.1
\end{tabular}

(*Los valores listados para el modelo son los obtenidos para las siguientes solicitaciones: Compresión: $1000 \mathrm{~N}$, Cortante: 100 N, Momentos (T,Fl.,Ex., F.L.): 6 Nm).

La Tabla 3 presenta un resumen de los distintos valores de rigidez media de los segmentos lumbares, obtenidos experimentalmente por diferentes autores ${ }^{9}$ 5,10,11,12, incluyendo los obtenidos con el presente modelo de elementos finitos para el segmento L3-L4. Los valores que se reflejan en la tabla corresponden a rigidez secante en cada una de las direcciones de solicitación anteriormente citadas, esto es, para las fuerzas: cociente entre fuerza aplicada y desplazamiento del 
centro del cuerpo L3 en la dirección de la solicitación y para los momentos: cociente entre el par aplicado y el giro producido en su misma dirección. Debido a la no linealidad del modelo, la rigidez secante dependerá de la magnitud de la solicitación aplicada, siendo los valores presentados en la Tabla 3 los obtenidos, por el modelo, para las siguientes excitaciones: Compresión: $1000 \mathrm{~N}$, Cortante: 100 N, Torsión, Flexión, Extensión y Flexión lateral: $6 \mathrm{Nm}$., los cuales corresponden a unos valores medios de los utilizados por los distintos autores referenciados.

Estos valores de rigidez secante deben considerarse como cifras orientativas que permiten conocer el orden de magnitud de las mismas, debido a las diferentes condiciones experimentales utilizadas por los autores: distinta ubicación y magnitud en las excitaciones, distintos segmentos lumbares, distintas dimensiones y condiciones de los discos intervertebrales, etc.

Como puede comprobarse los valores obtenidos con el modelo resultan coherentes con los encontrados en la literatura. En el caso de solicitación de torsión encontramos un valor ligeramente inferior que el resto de los autores referenciados, aunque otros autore ${ }^{14,15}$ han encontrado valores inferiores. Por otro lado se ha estudiado también la influencia de una precompresión (400 N aplicados sobre el centro del cuerpo vertebral L3, valor utilizado por Schultz y $\mathrm{cols}^{12}$ ) en el comportamiento a torsión del segmento L3-L4, los resultados obtenidos aparecen en la Figura 10, pudiendo apreciarse un aumento de la rigidez a torsión del segmento en concordancia con lo encontrado por otros autores ${ }^{1,16}$, en concreto, para un valor del momento torsor de $6 \mathrm{Nm}$ encontramos un valor de rigidez secante a torsión de $6.9 \mathrm{Nm} /{ }^{\circ}$

Para las solicitaciones rasantes, en ausencia de compresión, podemos observar un comportamiento similar del segmento ante cortante anterior y posterior para valores de solicitación menores a 100 $\mathrm{N}$, aumentando, a partir de ese valor, la rigidez ante fuerza anterior respecto a la correspondiente a fuerza posterior, debido al establecimiento del contacto entre los elementos Gap de las carillas articulares. Por ello en la tabla 3 aparece un valor ligeramente inferior para la rigidez a cortante anterior con solicitación de $100 \mathrm{~N}$, si se considerara la solicitación a $200 \mathrm{~N}$ se encontraría un valor de rigidez secante igual a $187 \mathrm{~N} / \mathrm{mm}$ para cortante anterior y $157 \mathrm{~N} / \mathrm{mm}$ para cortante posterior.

En cuanto a las solicitaciones de flexión, extensión y flexión lateral se obtuvieron valores de rigidez dentro del orden de magnitud de lo encontrado en la literatura, destacando que la mayor rigidez, para cualquier valor de los pares aplicados, corresponde a la solicitación de extensión.

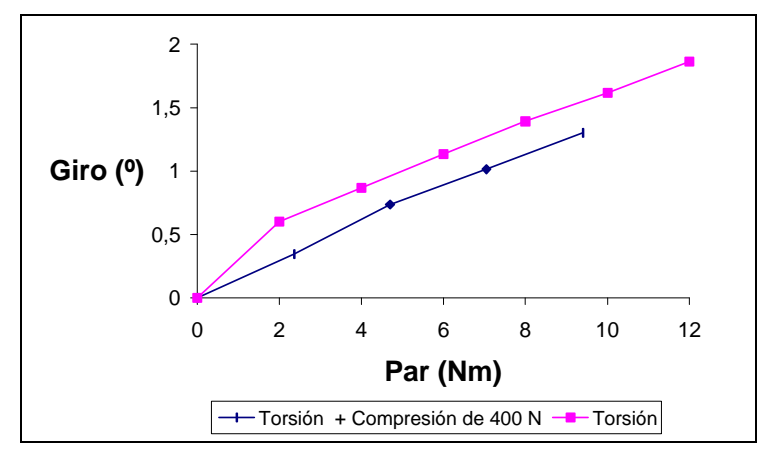

Figura 10. Giro de L3 vs Par torsor aplicado, con y sin compresión de $40 \mathrm{~N}$

\section{Columna lumbar completa}

Se ha sometido al modelo de la columna lumbar completa a una compresión variable entre 0 y 700 Newton, en las condiciones indicadas anteriormente, midiendose los deplazamientos de los centros de cada uno de los cuerpos vertebrales en la dirección perpendicular al plano medio del disco intervertebral L3-L4. Los resultados aparecen en la figura 11.

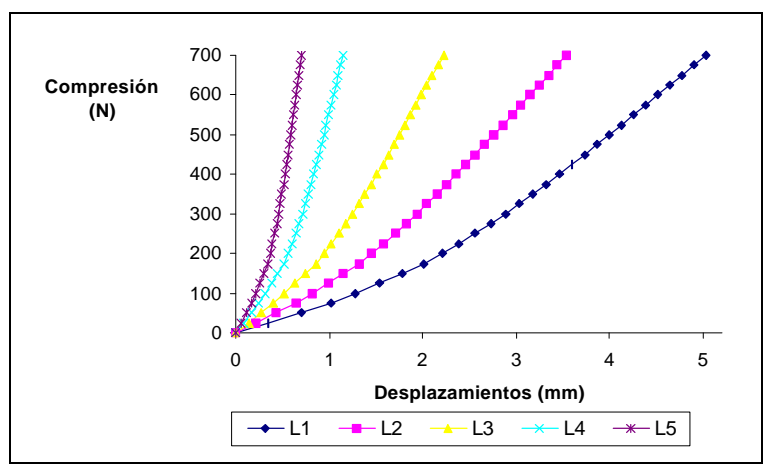

Figura 11. Desplazamientos axiales de las vértebras lumbares respecto a la fuerza de compresión aplicada, con el centro de L1 fijo en el plano XZ

La comparación con los resultados obtenidos por Shirazi-Adl y cols ${ }^{4}$ es favorable, encontrando tan solo pequeñas diferencias achacables a la distinta geometría de partida utilizada en uno y otro modelo. Cabe destacar que los resultados obtenidos con el modelo no solo predicen el nivel de deformación ante la carga máxima, $700 \mathrm{~N}$, sino que 
también revelan un aumento de rigidez de la columna lumbar, conforme se aumenta la compresión, similar al mostrado por el modelo de Shirazi-Adl y cols ${ }^{4}$.

\section{Conclusiones}

Se ha obtenido la respuesta del modelo ante diversas solicitaciones, pudiendo comprobar la similitud de la misma tanto respecto a resultados experimentales como respecto a otro modelo de Elementos Finitos. Por tanto parece que el modelo presentado refleja el comportamiento mecánico de la columna lumbar humana y por ello será posible su utilización en el objetivo, previamente expuesto, de utilizarse como herramienta de investigación aplicada a la cirugía ortopédica de columna lumbar.

\section{Bibliografía}

1. Belytschko T, Kulak RF, Schultz AB y Galante JO. Finite Element stress analysis of an intervertebral disc. J. Biomech. 21 (1), 59-66, 1974.

2. Shirazi-Adl A, Ahmed AM, Shrivastava SC. Mechanical response of a lumbar motion segment in axial torque alone and combined with compression. Spine 11 (9) 914-927, 1986.

3. Goel V.; Weizeng K.; Han J.; Weinstein J.; Gilbertson L. A combined finite element and optimization investigation of lumbar spine mechanics with and without muscles. Spine 18 (11), 1993

4. Shirazi-Adl A y Parnianpour M. Nonlinear response analisys of the human ligamentous lumbar spine in compression. Spine 18 (1). 1993.

5. Lavaste F, Skalli W, Robin S, Camille R, Mazel C. 3D geometrical and mechanical modelling of the lumbar spine. J. Biomech. 25 (10): 1153-1166, 1992.

6. Goel VK, Weinstein J. Biomechanics of the spine: Clinical and surgical perspective. Boca
Raton, CRC Press Inc., 1990.

7. White AA, Panjabi MM. Clinical biomechanics of the spine. J.B. Lippincott, Filadelfia, 1990.

8. Wu HC, Yao RF. Mechanical behaviour of the human annulus fibrosus. J. Biomech. 9,1-7, 1976.

9. Berkson MH, Nachemson A y Schultz AB. Mechanical properties of human lumbar spine motion segment- Part II: Responses in compression and shear: Influence of gross morphology. J. Biomech. Eng. 101, 53-57, 1979.

10. Markolf KL. Deformation of the thoracolumbar intervertebral joints in response to external loads. J. Bone Joint Surg [Am] 54, 511-533, 1971.

11. Panjabi MM, Krag MH, Chung TQ. Effects of disk injury on mechanical behavior of the human spine. Spine 9, 707-713. 1984.

12. Schultz AB, Warwick DN, Berkson MH, Nachemson A. Mechanical properties of human lumbar spine motion segment- Part I: Responses in flexion, extension, lateral bending and torsion. J. Biomech. Eng. 101, 4652, 1979.

13. Panjabi MM, Goel V, Oxland T, Takata K, Duranceau J, Price M. Human lumbar vertebrae: Quantitative three-dimensional anatomy. Spine 17 (3), 299-306. 1992.

14. Farfan MF, Cossette JW, Robertson GH, Wells $\mathrm{RV}$, Kraus $\mathrm{H}$. The effects of torsion on the intervertebral joints: The role of torsion in the production of disc degeneration. J. Bone Joint Surg. 25a . 468-497, 1970.

15. McGlashen KM, Miller JAA, Schultz AB, Andersson GBJ. Load displacement behavior of the human lumbo-sacral joint. J. Orthop. Res, 5, 488-496. 1987.

16. Skalli W, Robin S, Lavaste F, Dubousset J. A biomechanical analysis of short segment spinal fixation using a three- dimensional geometric and mechanical model. Spine, 18 (5), 536545.1993 